Isomorphism classes for Banach vector bundle structures of second tangents

Dodson, CTJ and Galanis, GN and Vassiliou, E 2005

MIMS EPrint: 2005.47

Manchester Institute for Mathematical Sciences

School of Mathematics

The University of Manchester

\footnotetext{
Reports available from: http://eprints.maths.manchester.ac.uk/

And by contacting: The MIMS Secretary

School of Mathematics

The University of Manchester

Manchester, M13 9PL, UK
} 


\title{
ISOMORPHISM CLASSES FOR BANACH VECTOR BUNDLE STRUCTURES OF SECOND TANGENTS
}

\author{
C.T.J. DODSON, G.N. GALANIS, AND E. VASSILIOU
}

\begin{abstract}
On a smooth Banach manifold $M$, the equivalence classes of curves that agree up to acceleration form the second order tangent bundle $T^{2} M$ of $M$. This is a vector bundle in the presence of a linear connection $\nabla$ on $M$ and the corresponding local structure is heavily dependent on the choice of $\nabla$. In this paper we study the extent of this dependence and we prove that it is closely related to the notions of conjugate connections and second order differentials. In particular, the vector bundle structure on $T^{2} M$ remains invariant under conjugate connections with respect to diffeomorphisms of $M$.
\end{abstract}

\section{INTRODUCTION}

The fibre bundle $T^{2} M$ of second order tangents to a smooth manifold $M$ arises in a natural way in several problems of theoretical physics and differential geometry (cf., for instance, [5], [7]). We recall that $T^{2} M$ consists of all equivalence classes of curves in $M$ that agree up to their acceleration. However, the definition of a vector bundle structure on $T^{2} M$ is not as straightforward as that of the first order tangent bundle $T M$ of $M$.

In [7], Dodson and Radivoiovici studied finite-dimensional second order tangent bundles and identified conditions under which these bundles admit the structure of a vector bundle. Dodson and Galanis ([4]) extended that study to the infinite dimensional case, namely to tangent bundles of order two over Banach and Fréchet manifolds (see also Section 1). In all these cases, existence of a vector bundle structure on $T^{2} M$ relies heavily on the choice of a linear connection $\nabla$ on the base manifold $M$; the trivializations are directly defined by the Christoffel symbols of $\nabla$. Indeed, every linear connection on $M$ determines one isomorphism $T^{2} M \equiv$ $T M \oplus T M,([7])$. Then it is natural to ask how these individual isomorphisms may be classified.

The answer to this question naturally leads us to the definition of the second order differential $T^{2} f$ of a smooth map $f: M \rightarrow N$ between two manifolds $M$ and $N$. In contrast to the case of the first order differential $T f$, the linearity of $T^{2} f$ on the fibres $\left(T_{x}^{2} f: T_{x}^{2} M \rightarrow T_{f(x)}^{2} N, x \in M\right)$ is not always ensured. However, we prove the following in Section 2:

A sufficient condition for linearity of $T_{x}^{2} f$ is that the linear connections $\nabla_{M}, \nabla_{N}$ inducing the structures of $T^{2} M$ and $T^{2} N$ respectively, are $f$-conjugate;

that is, roughly speaking, the connections commute with the differentials of $f$. Furthermore, in that case, $T^{2} f$ becomes a vector bundle morphism. This conjugacy result allows us to classify, in a sense, the structure of $T^{2} M$, thus answering the initial question in the following manner:

The vector bundle structure of $T^{2} M$ defined by $\nabla$, remains invariant (isomorphic) if $\nabla$ is replaced by an $f$-conjugate connection, for any diffeomorphism $f$ of $M$.

Date: 13 October 2005

2000 Mathematical Subject Classification. Primary 58B25; Secondary 58A05. 
Our approach covers the case of Banach manifolds and bundles, and is susceptible to further generalization to any infinite-dimensional manifold admitting appropriate linear connections.

\section{Preliminaries}

In this section we present the necessary material needed in the sequel. Let $M$ be a smooth manifold modeled on a Banach space $\mathbb{E}$ and $\left\{\left(U_{\alpha}, \phi_{\alpha}\right)\right\}_{\alpha \in I}$ a corresponding atlas. Then, the tangent bundle $T M$ of $M$ admits the local coordinate system $\left\{\left(\pi_{M}^{-1}\left(U_{\alpha}\right), \tau_{\alpha}\right)\right\}_{\alpha \in I}$ with

$$
\tau_{\alpha}: \pi_{M}^{-1}\left(U_{\alpha}\right) \longrightarrow \phi_{\alpha}\left(U_{\alpha}\right) \times \mathbb{E}:[(c, x)] \mapsto\left(\phi_{\alpha}(x),\left(\phi_{\alpha} \circ c\right)^{\prime}(0)\right),
$$

where $[(c, x)]$ stands for the equivalence class of a smooth curve $c$ of $M$ with $c(0)=x$ and $\left(\phi_{\alpha} \circ c\right)^{\prime}(0)=\left[d\left(\phi_{\alpha} \circ c\right)(0)\right](1)$. A trivialization $\left\{\left(\pi_{T M}^{-1}\left(\pi_{M}^{-1}\left(U_{\alpha}\right)\right), \widetilde{\tau}_{\alpha}\right)\right\}_{\alpha \in I}$ of $T(T M)$ is analogously defined.

A vector bundle morphism

$$
\nabla: T(T M) \longrightarrow T M
$$

with local expressions, relative to the above charts,

$$
\nabla_{\alpha}:=\tau_{\alpha} \circ \nabla \circ\left(\widetilde{\tau}_{\alpha}\right)^{-1}: \phi_{\alpha}\left(U_{\alpha}\right) \times \mathbb{E} \times \mathbb{E} \times \mathbb{E} \longrightarrow \phi_{\alpha}\left(U_{\alpha}\right) \times \mathbb{E},
$$

is called a linear connection of $M$ if the maps $\omega_{\alpha}: \phi_{\alpha}\left(U_{\alpha}\right) \times \mathbb{E} \rightarrow \mathcal{L}(\mathbb{E})$, defined by the relations

$$
\nabla_{\alpha}(y, u, v, w)=\left(y, w+\omega_{\alpha}(y, u) \cdot v\right), \quad a \in I,
$$

are smooth, and linear with respect to the second variable. Here $\mathcal{L}(\mathbb{E})$ stands for the space of continuous linear maps of $\mathbb{E}$.

On the other hand, a connection $\nabla$ is completely determined by the family of its Christoffel symbols

$$
\Gamma_{\alpha}: \phi_{\alpha}\left(U_{\alpha}\right) \longrightarrow \mathcal{L}(\mathbb{E}, \mathcal{L}(\mathbb{E}, \mathbb{E})) ; \quad \alpha \in I,
$$

defined by

$$
\Gamma_{\alpha}(y)(u)=\omega_{\alpha}(y, u),(y, u) \in \phi_{\alpha}\left(U_{\alpha}\right) \times \mathbb{E} .
$$

The latter satisfy also the following compatibility condition:

$$
\begin{gathered}
\Gamma_{\beta}\left(\phi_{\beta \alpha}(y)\right)\left(D \phi_{\beta \alpha}(y)(u)\right)\left(D \phi_{\beta \alpha}(y)(v)\right)+\left(D^{2} \phi_{\beta \alpha}(y)(v)\right)(u)= \\
=D \phi_{\beta \alpha}(y)\left(\Gamma_{\alpha}(y)(u)(v)\right),
\end{gathered}
$$

for every $(y, u, v) \in \phi_{\alpha}\left(U_{\alpha} \cap U_{\beta}\right) \times \mathbb{E} \times \mathbb{E}$ and $\phi_{\beta \alpha}:=\phi_{\beta} \circ \phi_{\alpha}^{-1}$. For details on this, coordinate free, approach and the relevant proofs we refer to [8], [17].

The presence of a linear connection on the manifold $M$ is the crucial property ensuring the existence of a vector bundle structure on the second order tangent bundle, $T^{2} M$, which is the fibre bundle

$$
T^{2} M:=\bigcup_{x \in M} T_{x}^{2} M
$$

Here $T_{x}^{2} M:=C_{x} / \approx_{x}$ denotes the tangent space of order two over $x$, with $C_{x}$ the set of all smooth curves in $M$ through $x$, and the equivalence relation $\approx_{x}$ on $C_{x}$ given by

$$
c_{1} \approx_{x} c_{2} \Longleftrightarrow c_{1}(0)=c_{2}(0)=x, \quad c_{1}^{\prime}(0)=c_{2}^{\prime}(0) \quad \text { and } \quad c_{1}^{\prime \prime}(0)=c_{2}^{\prime \prime}(0) .
$$

Denote by $\pi_{M}^{2}$ the natural projection:

$$
\pi_{M}^{2}: T^{2} M \longrightarrow M:[(c, x)]_{2} \mapsto x,
$$

where $[(c, x)]_{2}$ is the equivalence class of the smooth curve $c \in C_{x}$, then the local trivializations

$$
\Phi_{\alpha}:\left(\pi_{M}^{2}\right)^{-1}\left(U_{\alpha}\right) \longrightarrow U_{\alpha} \times \mathbb{E} \times \mathbb{E}
$$


of $T^{2} M$ arise from the original smooth atlas $\left\{\left(U_{\alpha}, \phi_{\alpha}\right)\right\}_{\alpha \in I}$ of $M$ by setting:

$\Phi_{\alpha}\left([(c, x)]_{2}\right)=\left(x,\left(\phi_{\alpha} \circ c\right)^{\prime}(0),\left(\phi_{\alpha} \circ c\right)^{\prime \prime}(0)+\Gamma_{\alpha}\left(\phi_{\alpha}(x)\right)\left(\left(\phi_{\alpha} \circ c\right)^{\prime}(0)\right)\left(\left(\phi_{\alpha} \circ c\right)^{\prime}(0)\right)\right)$

In this way, $T^{2} M$ turns out to be a vector bundle over $M$ with fibres of type $\mathbb{E} \times \mathbb{E}$ and structure group $G L(\mathbb{E} \times \mathbb{E})$. If $\Phi_{\alpha, x}$ denotes the restriction of the trivializing map $\Phi_{\alpha}$ on the fibre $T_{x}^{2} M$, then the corresponding transition functions

$$
T_{\alpha \beta}: U_{\alpha} \cap U_{\beta} \longrightarrow \mathcal{L}(\mathbb{E} \times \mathbb{E}, \mathbb{E} \times \mathbb{E}): x \mapsto \Phi_{\alpha, x} \circ \Phi_{\beta, x}^{-1}
$$

are given by

$$
T_{\alpha \beta}=\left(D \phi_{\alpha \beta} \circ \phi_{\beta}\right) \times\left(D \phi_{\alpha \beta} \circ \phi_{\beta}\right) ; \quad \alpha, \beta \in I .
$$

\section{SECOND ORDER DIFFERENTIALS}

We proceed to the definition of second order differentials. To this end, we consider two smooth manifolds $M$ and $N$ modelled on the Banach spaces $\mathbb{E}$ and $\mathbb{F}$, respectively, with corresponding atlases $\mathcal{A}=\left\{\left(U_{\alpha}, \phi_{a}\right)\right\}_{\alpha \in I}$ and $\mathcal{B}=\left\{\left(V_{\beta}, \psi_{\beta}\right)\right\}_{\beta \in J}$. We fix two linear connections $\nabla_{M}, \nabla_{N}$ on $M, N$ respectively, with Christoffel symbols $\left\{\Gamma_{\alpha}^{M}\right\}_{\alpha \in I},\left\{\Gamma_{\beta}^{N}\right\}_{\beta \in J}$. As explained in Section 1, the pairs $\left(M, \nabla_{M}\right)$ and $\left(N, \nabla_{N}\right)$ induce the second order bundles $T^{2} M$ and $T^{2} N$ with vector bundle atlases $\mathcal{A}^{2}=\left\{\left(\left(\pi_{M}^{2}\right)^{-1}\left(U_{\alpha}\right), \Phi_{a}\right)\right\}_{\alpha \in I}$ and $\mathcal{B}^{2}=\left\{\left(\left(\pi_{N}^{2}\right)^{-1}\left(V_{\beta}\right), \Psi_{\beta}\right)\right\}_{\beta \in J}$.

With the previous assumptions we have the following.

Definition 2.1. If $g: M \rightarrow N$ is a smooth map, we call the second order differential of $g$ the map

$$
T^{2} g: T^{2} M \longrightarrow T^{2} N:[(c, x)]_{2} \mapsto[(g \circ c, g(x))]_{2} .
$$

$T^{2} g$ is a well defined map. Indeed, for two $\approx_{x}$-equivalent smooth curves $c_{1}, c_{2}$ of $M$ through $x$, we see that

$$
\begin{gathered}
\left(g \circ c_{1}\right)(0)=\left(g \circ c_{2}\right)(0)=g(x), \\
\left(g \circ c_{1}\right)^{\prime}(0)=T_{0}\left(g \circ c_{1}\right)(1)=T_{x} g\left(c_{1}^{\prime}(0)\right)=T_{x} g\left(c_{2}^{\prime}(0)\right)=\left(g \circ c_{2}\right)^{\prime}(0), \\
\left(g \circ c_{1}\right)^{\prime \prime}(0)=T_{c_{1}^{\prime}(0)}\left(T_{c_{1}(0)} g\right)\left(c_{1}^{\prime \prime}(0)\right)=T_{c_{2}^{\prime}(0)}\left(T_{c_{2}(0)} g\right)\left(c_{2}^{\prime \prime}(0)\right)=\left(g \circ c_{2}\right)^{\prime \prime}(0) .
\end{gathered}
$$

However, though always a fibre bundle morphism, the required linearity of $T^{2} g$ on the fibres of $T^{2} M$ and $T^{2} N$ is not always ensured, in contrast to the case of ordinary (first order) differentials. As a matter of fact, for every choice of vectors $(u, v) \in \mathbb{E} \times \mathbb{E}$, if we denote by $c$ the smooth curve of $M$ realizing $(u, v)$ via the trivialization $\left(U_{\alpha}, \Phi_{\alpha}\right)$ of $T^{2} M$, i.e.

$$
\begin{gathered}
(u, v)=\Phi_{\alpha, x}\left([(c, x)]_{2}\right) \\
=\left(\left(\phi_{\alpha} \circ c\right)^{\prime}(0),\left(\phi_{\alpha} \circ c\right)^{\prime \prime}(0)+\Gamma_{\alpha}^{M}\left(\phi_{\alpha}(x)\right)\left(\left(\phi_{\alpha} \circ c\right)^{\prime}(0)\right)\left(\left(\phi_{\alpha} \circ c\right)^{\prime}(0)\right)\right),
\end{gathered}
$$


and setting $G:=\psi_{\beta} \circ g \circ \phi_{\alpha}^{-1}$, then the corresponding local expression of $T_{x}^{2} g:=$ $\left.T^{2} g\right|_{T_{x}^{2} M}$ takes the following form:

$$
\begin{aligned}
& \left(\Psi_{\beta, g(x)} \circ T_{x}^{2} g \circ \Phi_{a, x}^{-1}\right)(u, v) \\
= & \left(\left(\psi_{\beta} \circ g \circ c\right)^{\prime}(0),\left(\psi_{\beta} \circ g \circ c\right)^{\prime \prime}(0)\right. \\
& \left.+\Gamma_{\beta}^{N}\left(\psi_{\beta}(g(x))\right)\left(\left(\psi_{\beta} \circ g \circ c\right)^{\prime}(0)\right)\left(\left(\psi_{\beta} \circ g \circ c\right)^{\prime}(0)\right)\right) \\
= & \left(D G\left(\phi_{\alpha}(x)\right)\left(\left(\phi_{\alpha} \circ c\right)^{\prime}(0)\right), D G\left(\phi_{\alpha}(x)\right)\left(\left(\phi_{\alpha} \circ c\right)^{\prime \prime}(0)\right)\right. \\
& +D^{2} G\left(\phi_{\alpha}(x)\right)\left(\left(\phi_{\alpha} \circ c\right)^{\prime}(0),\left(\phi_{\alpha} \circ c\right)^{\prime}(0)\right) \\
& \left.+\Gamma_{\beta}^{N}\left(\psi_{\beta}(g(x))\right)\left(D G\left(\phi_{\alpha}(x)\right)\left(\left(\phi_{\alpha} \circ c\right)^{\prime}(0)\right)\right)\left(D G\left(\phi_{\alpha}(x)\right)\left(\left(\phi_{\alpha} \circ c\right)^{\prime}(0)\right)\right)\right) \\
= & \left(D G\left(\phi_{\alpha}(x)\right)(u), D G\left(\phi_{\alpha}(x)\right)(v)-D G\left(\phi_{\alpha}(x)\right)\left(\Gamma_{\alpha}^{M}\left(\phi_{\alpha}(x)\right)(u)(u)\right)\right. \\
& \left.+D^{2} G\left(\phi_{\alpha}(x)\right)(u, u)+\Gamma_{\beta}^{N}\left(\psi_{\beta}(g(x))\right)\left(D G\left(\phi_{\alpha}(x)\right)(u)\right)\left(D G\left(\phi_{\alpha}(x)\right)(u)\right)\right) \\
= & \left(D G\left(\phi_{\alpha}(x)\right)(u), D G\left(\phi_{\alpha}(x)\right)(v)-\left(D G\left(\phi_{\alpha}(x)\right)\left(\Gamma_{\alpha}^{M}\left(\phi_{\alpha}(x)\right)(u)(u)\right)\right.\right. \\
& \left.+D^{2} G\left(\phi_{\alpha}(x)\right)(u, u)+\Gamma_{\beta}^{N}\left(G\left(\phi_{\alpha}(x)\right)\right)\left(D G\left(\phi_{\alpha}(x)\right)(u)\right)\left(D G\left(\phi_{\alpha}(x)\right)(u)\right)\right) .
\end{aligned}
$$

Obviously, the presence of the Christoffel symbols and the derivatives of second order, prevents $T_{x}^{2} g$ from being linear.

A way out of this drawback is obtained via the notion of conjugate connections. More precisely, with the previous notations, the connections $\nabla_{M}$ and $\nabla_{N}$ are called $g$-conjugate (or $g$-related) if they commute with the differentials of $g$, i.e.,

$$
T g \circ \nabla_{M}=\nabla_{N} \circ T(T g) \text {. }
$$

The local expression of the latter is the following:

$$
\begin{gathered}
D G\left(\phi_{\alpha}(x)\right)\left(\Gamma_{\alpha}^{M}\left(\phi_{\alpha}(x)\right)(u)(u)\right)= \\
\Gamma_{\beta}^{N}\left(G\left(\phi_{\alpha}(x)\right)\right)\left(D G\left(\phi_{\alpha}(x)\right)(u)\right)\left(D G\left(\phi_{\alpha}(x)\right)(u)\right)+D(D G)\left(\left(\phi_{\alpha}(x)\right)(u, u),\right.
\end{gathered}
$$

for every $(x, u) \in U_{\alpha} \times \mathbb{E}$ (for details on conjugate connections see [16]).

Taking now into account (2), we may check that a sufficient condition leading to the elimination of the non linear part of $T_{x}^{2} g$ is the conjugation relationship of the connections $\nabla_{M}$ and $\nabla_{N}$ in use. Indeed, in this case the local expression of $T_{x}^{2} g$ reduces to

$$
\left(\Psi_{\beta, g(x)} \circ T_{x}^{2} g \circ \Phi_{a, x}^{-1}\right)(u, v)=\left(D G\left(\phi_{\alpha}(x)\right)(u), D G\left(\phi_{\alpha}(x)\right)(v)\right) .
$$

Therefore, one infers the following.

Proposition 2.2. The second order differential $T^{2} g: T^{2} M \rightarrow T^{2} N$ is linear on the fibres if the connections $\nabla_{M}$ and $\nabla_{N}$ are g-conjugate.

Remark 2.3. The conjugacy relationship employed in Proposition 2.2 is a sufficient, but not necessary condition ensuring the linearity of $T^{2} g$. The optimal (necessary and sufficient) choice would be to assume that the 'problematic' part

$$
\begin{aligned}
\mathbb{E} \ni u \longmapsto & -D G\left(\phi_{\alpha}(x)\right)\left(\Gamma_{\alpha}^{M}\left(\phi_{\alpha}(x)\right)(u)(u)\right)+D^{2} G\left(\phi_{\alpha}(x)\right)(u, u) \\
& \left.+\Gamma_{\beta}^{N}\left(G\left(\phi_{\alpha}(x)\right)\right)\left(D G\left(\phi_{\alpha}(x)\right)(u)\right)\left(D G\left(\phi_{\alpha}(x)\right)(u)\right)\right) \in \mathbb{E}
\end{aligned}
$$

appearing in the local expression of $T_{x}^{2} g$, is a linear, not necessarily zero, map. There is ongoing research into the possible geometric consequences of such an assumption.

Example 2.4. We give here two examples of conjugacy classes in order to clarify somewhat our construction.

1. In the case of a constant map $g$, equality (2) collapses to a trivial identification of zero quantities, since the local expression $G$ becomes also constant. As a result, all linear connections are conjugate through constant maps. 
2. If we consider the identity map $g=i d_{M}$, then equality (2) takes the form

$$
\begin{gathered}
D \phi_{\beta \alpha}\left(\phi_{\alpha}(x)\right)\left(\Gamma_{\alpha}^{M}\left(\phi_{\alpha}(x)\right)(u)(u)\right)= \\
\Gamma_{\beta}^{N}\left(\phi_{\beta}(x)\right)\left(D \phi_{\beta \alpha}\left(\phi_{\alpha}(x)\right)(u)\right)\left(D \phi_{\beta \alpha}\left(\phi_{\alpha}(x)\right)(u)\right)+D^{2} \phi_{\beta \alpha}\left(\phi_{\alpha}(x)\right)(u, u) .
\end{gathered}
$$

The latter is equivalent to the compatibility condition satisfied by the Christoffel symbols of a connection on $M$ (see Preliminary Section). Note that the previous relation holds also for non-diagonal elements $(u, v)$ as we easily may check due to the bilinearity of the involved functions. As a result, any $i d_{M}$-conjugate connections have to be equal and the conjugation relationship in this case reduces to equality.

Proposition 2.2 leads to the following main result.

Theorem 2.5. Let $T^{2} M, T^{2} N$ be the second order tangent bundles defined by the pairs $\left(M, \nabla_{M}\right),\left(N, \nabla_{N}\right)$, and let $g: M \rightarrow N$ be a smooth map. If the connections $\nabla_{M}$ and $\nabla_{N}$ are $g$-conjugate, then the second order differential $T^{2} g: T^{2} M \rightarrow T^{2} N$ is a vector bundle morphism.

Proof. It is easy to check that the pair $\left(g, T^{2} g\right)$ commutes with the natural projections of the second order tangent bundles, since $T^{2} g$ is fibre preserving. As a result, in virtue of Proposition 2.2, it remains to check the local behaviour of $T^{2} g$ with respect to the trivializations $\left(U_{\alpha}, \Phi_{\alpha}\right)$ of $T^{2} M$ and $\left(V_{\beta}, \Psi_{\beta}\right)$ of $T^{2} N$. By equality (3), we see that the map

$$
U_{\alpha} \longrightarrow \mathcal{L}(\mathbb{E} \times \mathbb{E}, \mathbb{F} \times \mathbb{F}): x \mapsto \Psi_{\beta, g(x)} \circ T_{x}^{2} g \circ \Phi_{a, x}^{-1}
$$

becomes smooth as coinciding with $D G\left(\phi_{\alpha}(x)\right) \times D G\left(\phi_{\alpha}(x)\right)$. Here $G$ denotes, once again, the local expression of $g$ with respect to the charts $\phi_{a}, \psi_{\beta}$. Hence, all the conditions of a vector bundle morphism (see, e.g., [11]) are fulfilled.

Remark 2.6. If we drop the conjugation assumption for $\nabla_{M}, \nabla_{N}$, then the local expression of $T^{2} g$ is

$$
\begin{aligned}
& \left(\Psi_{\beta} \circ T^{2} g \circ \Phi_{a}^{-1}\right)(x, u, v)= \\
& =\left(D G\left(\phi_{\alpha}(x)\right)(u), D G\left(\phi_{\alpha}(x)\right)(v)-D G\left(\phi_{\alpha}(x)\right)\left(\Gamma_{\alpha}^{M}\left(\phi_{\alpha}(x)\right)\right)(u)(u)\right. \\
& \left.+D^{2} G\left(\phi_{\alpha}(x)\right)(u, u)+\Gamma_{\beta}^{N}\left(G\left(\phi_{\alpha}(x)\right)\right)\left(D G\left(\phi_{\alpha}(x)\right)(u)\right)\left(D G\left(\phi_{\alpha}(x)\right)(u)\right)\right) .
\end{aligned}
$$

This implies that $T^{2} g$ is a smooth map and $\left(g, T^{2} g\right)$ is a fibre (but not necessarily vector) bundle morphism.

\section{Connection Dependence of SECOnd order StruCtures}

As we have already seen, the vector bundle structure of $T^{2} M$ depends heavily on the choice of a linear connection $\nabla$ on the base manifold $M$. With the results of the preceding section, we are now in a position to discuss the extent of this dependence.

Precisely, we obtain:

Theorem 3.1. Let $\nabla, \nabla^{\prime}$ be two linear connections on $M$. If $g$ is a diffeomorphism of $M$ such that $\nabla$ and $\nabla^{\prime}$ are $g$-conjugate, then the vector bundle structures on $T^{2} M$, induced by $\nabla$ and $\nabla^{\prime}$, are isomorphic.

The proof is an obvious application of Theorem 2.5

We introduce the following terminology :

Two pairs $(M, \nabla)$ and $\left(M, \nabla^{\prime}\right)$, where the linear connections $\nabla, \nabla^{\prime}$ are $g$ conjugate with respect to a diffeomorphism $g$ of $M$, as in Theorem 3.1, are called equivalent. Then, Theorem 3.1 implies that: 
Corollary 3.2. Up to isomorphism, the elements of the g-conjugate equivalence class $[(M, \nabla)]_{g}$ determine the same vector bundle structure on $T^{2} M$. Consequently, the latter structure depends not only on a pair $(M, \nabla)$ but also on the entire class $[(M, \nabla)]_{g}$.

From the previous discussion, it becomes clear now that the identification of two bundles $T^{2} M, T^{2} N$ is not ensured by the existence of a mere diffeomorphism $g: M \stackrel{\cong}{\longrightarrow} N$. One has to take into account the geometry of $M$ and $N$, expressed by the linear connections on them.

Another point of view of the totality of linear connections on a smooth finite dimensional manifold $M$ worth noting here is that of system of connections devised by Mangiarotti and Modugno $([12,13])$. Namely, whereas the function space of all linear connections is infinite dimensional even in the case of finite dimensional $M$, it is possible to obtain a finite dimensional bundle-representation of all linear connections on $M$ in terms of such a connection system. Indeed, there exists a unique universal connection of which every connection in the system of connections is a pullback. A similar relation holds between the corresponding universal curvature and the curvatures of the connections of the system. This is a different representation of an object similar to that introduced by Narasimhan and Ramanan $[14,15]$ for $G$-bundles, also allowing a proof of Weil's theorem (cf. $[10,9,2])$.

The system of all linear connections on a finite dimensional manifold $M$ has a representation on the tangent bundle via the system space

$$
C_{T}=\left\{\alpha \otimes j \gamma \in T^{*} M \otimes_{M} J T M \mid j \gamma: T M \rightarrow T T M \text { projects onto } I_{T M}\right\} .
$$

Here we view $I_{T M}$ as a section of $T^{*} M \otimes T M$, which is a subbundle of $T^{*} M \otimes$ $T T M$, with local expression $d x^{\lambda} \otimes \partial_{\lambda}$.

The fibred morphism for the system $C_{T}$ is

$$
\begin{aligned}
\xi_{T}: C_{T} \times_{M} T M & \longrightarrow J T M \subset T^{*} M \otimes_{T M} T T M, \\
(\alpha \otimes j \gamma, \nu) & \longmapsto \alpha(\nu) j \gamma .
\end{aligned}
$$

In coordinates $\left(x^{\lambda}\right)$ on $M$ and $\left(y^{\lambda}\right)$ on $T M$

$$
\xi_{T}=d x^{\lambda} \otimes\left(\partial_{\lambda}-\gamma_{\lambda}^{i} \partial_{i}\right)=d x^{\lambda} \otimes\left(\partial_{\lambda}-y^{j} \Gamma_{j \lambda}^{i} \partial_{i}\right) .
$$

Each section of $C_{T} \rightarrow M$, such as $\tilde{\Gamma}: M \rightarrow C_{T}:\left(x^{\lambda}\right) \rightarrow\left(x^{\lambda}, \gamma_{\mu \vartheta}\right)$; determines the unique linear connection $\Gamma=\xi_{T} \circ\left(\tilde{\Gamma} \circ \pi_{T}, I_{T M}\right)$ with Christoffel symbols $\Gamma_{\mu \vartheta}^{\lambda}$.

On the fibred manifold $\pi_{1}: C_{T} \times_{M} T M \rightarrow C_{T}$; the universal connection is given by:

$$
\begin{aligned}
& \Lambda_{T}: C_{T} \times_{M} T M \longrightarrow J\left(C_{T} \times_{M} T M\right) \subset T^{*} C_{T} \otimes T\left(C_{T} \times_{M} T M\right) \\
& \left(x^{\lambda}, v_{\mu \nu}^{\lambda}, y^{\lambda}\right) \longmapsto\left[\left(X^{\lambda}, V_{\mu \nu}^{\lambda}\right) \rightarrow\left(X^{\lambda}, V_{\mu \nu}^{\lambda}, Y^{\mu} V_{\mu \nu}^{\lambda} X^{\nu}\right)\right] .
\end{aligned}
$$

In coordinates,

$$
\Lambda_{T}=d x^{\lambda} \otimes \partial_{\lambda}+d v^{a} \otimes \partial_{a}+y^{\mu} v_{\mu \nu}^{i} d x^{\nu} \otimes \partial_{i} .
$$

Explicitly, each $\tilde{\Gamma} \in \operatorname{Sec}\left(C_{T} / M\right)$ gives an injection $\left(\tilde{\Gamma} \circ \pi_{T}, I_{T M}\right)$, of $T M$ into $C_{T} \times T M$, which is a section of $\pi_{1}, \Gamma$ coincides with the restriction of $\Lambda_{T}$ to this section:

$$
\Lambda_{T \mid\left(\tilde{\Gamma} \circ \pi_{T}, I_{T M}\right) T M}=\Gamma
$$

and the universal curvature of the connection $\Lambda$ is given by:

$$
\Omega_{T}=d_{\Lambda_{T}} \Lambda_{T}: C_{T} \times_{M} T M \rightarrow \wedge^{2}\left(T^{*} C_{T}\right) \otimes_{T M} V(T M) .
$$

So, here the universal curvature $\Omega_{T}$ has the coordinate expression:

$$
\Omega_{T}=\frac{1}{2}\left(y^{k} v_{k \lambda}^{j} \partial_{j} y^{m} v_{m \mu}^{i} d x^{\lambda} \wedge d x^{\mu}+2 \partial_{a} y^{m} v_{m \mu}^{i} d x^{a} \wedge d x^{\mu}\right) \otimes \partial_{i} .
$$


For more details of the corresponding universal calculus see Dodson and Modugno [6]. In the case of Riemannian and pseudo-Riemannian manifolds, Canarutto and Dodson [1] used systems of principal connections to establish certain incompleteness stability properties; Del Riego and Dodson [3] established certain topological and universal properties of sprays and Lie algebras, obtaining associated completeness criteria.

The system of linear connections provides a bundle framework in which choices of linear connection may be made, and hence vector bundle structures on $T^{2} M$ are determined. It would be interesting to extend to infinite dimensional Banach manifolds the systems of connections approach using the methodology presented in this paper, so as to characterize further the isomorphism classes of second order tangents.

Acknowledgement The third author has been partially supported by the Special Account Research Grant 70/4/3410, University of Athens.

\section{REFERENCES}

[1] D. Canarutto and C.T.J. Dodson. On the bundle of principal connections and the stability of b-incompleteness of manifolds. Math. Proc. Camb. Phil. Soc. 98 (1985), 51-59.

[2] L.A. Cordero, C.T.J. Dodson and M. de LEON. Differential Geometry of Frame Bundles. (Kluwer, Dordrecht, 1989).

[3] L. Del Riego and C.T.J. Dodson. Sprays, universality and stability. Math. Proc. Camb. Phil. Soc. 103 (1988), 515-534.

[4] C.T.J. Dodson and G.N. Galanis. Second order tangent bundles of infinite dimensional manifolds. J. Geom. Phys. 52 (2004), 127-136.

[5] C.T.J. Dodson and G.N. Galanis. Bundles of acceleration on Banach manifolds. Nonlinear Analysis 63, 5-7 (2005) 465-471.

[6] C.T.J. Dodson and M. Modugno. Connections over connections and universal calculus. In Proc. VI Convegno Nazionale di Relativita General e Fisica Della Gravitazione Florence, 1013 October 1984, Eds. R. Fabbri and M. Modugno, pp. 89-97, Pitagora Editrice, Bologna, 1986.

[7] C.T.J. Dodson and M.S. Radivoiovici. Tangent and Frame bundles of order two. Anal. Stiint. Univ. "Al. I. Cuza" 28, (1982) 63-71.

[8] P. Flaschel and W. Klingenberg, Riemannsche Hilbertmannigfaltigkeiten Periodische Geodätische. Lecture Notes in Math. 282 (Springer-Verlag, Berlin, 1972).

[9] P.L. Garcia. Connections and 1-jet fibre bundles. Rend. Sem. Mat. Univ. Padova. 47 (1972), 227-242.

[10] S. Kobayashi and K. Nomizu, Foundations of differential geometry Vol. II. (Interscience, New York, 1969).

[11] S. Lang, Differential manifolds. (Addison-Wesley, Reading Massachusetts, 1972).

[12] L. Mangiarotti and M. Modugno. Fibred spaces, jet spaces and connections for field theories. In Proc. International Meeting on Geometry and Physics, Florence, 12-15 October 1982, Ed. M. Modugno, Pitagora Editrice, Bologna, 1983, 135-165.

[13] M. Modugno. Systems of vector valued forms on a fibred manifold and applications to gauge theories. In Proc. Conference Differential Geomeometric Methods in Mathematical Physics, Salamanca 1985, Lecture Notes in Mathematics 1251 (Springer-Verlag, Berlin 1987, 238264).

[14] M.S. Narasimhan and S. Ramanan. Existence of universal connections I. Amer. J. Math. 83 (1961), 563-572.

[15] M.S. Narasimhan and S. Ramanan. Existence of universal connections II. Amer. J. Math. 85 (1963), 223-231.

[16] E. Vassiliou. Transformations of linear connections. Period. Math. Hungar. 13 (1982), 289308.

[17] J. Vilms. Connections on tangent bundles. J. Diff. Geom. 41 (1967), 235-243. 
School of Mathematics Manchester University, Manchester, M60 1QD, United KingDOM

E-mail address: ctdodson@manchester.ac.uk

Section of Mathematics, Naval Academy of Greece, Xatzikyriakion, Piraeus 18539 , GREECE

E-mail address: ggalanis@snd.edu.gr

Department of Mathematics, University of Athens, Panepistimiopolis, Athens 15784 , GREECE

E-mail address: evassil@cc.uoa.gr 